\title{
The Relationship Between Interpersonal Relationship and Math Achievement of Students with Poor Academic Performance
}

\author{
Zheng Haoyue, Luan Xiao Jiao, Wang Haitao* \\ Teaching Center of Fundamental Courses, Ocean University of China, Qingdao, China \\ Email address: \\ 793744056@qq.com (Zheng Haoyue), 1982824256@qq.com (Luan Xiao Jiao), yshdwht@163.com (Wang Haitao) \\ ${ }^{*}$ Corresponding author
}

\section{To cite this article:}

Zheng Haoyue, Luan Xiao Jiao, Wang Haitao. The Relationship Between Interpersonal Relationship and Math Achievement of Students with Poor Academic Performance. Science Innovation. Vol. 9, No. 3, 2021, pp. 92-95. doi: 10.11648/j.si.20210903.13

Received: March 12, 2021; Accepted: May 17, 2021; Published: May 24, 2021

\begin{abstract}
Interpersonal relationship has become an important aspect of social research on students with academic disabilities, because interpersonal relationship not only has an impact on the academic development of students with academic disabilities, but also causes a series of psychological problems, so it is of great theoretical and practical value to discuss this aspect. In order to explore the relationship between interpersonal relationship and math achievement of students with academic disability, this study adopted the method of cluster random sampling, using interpersonal relationship scale of primary and secondary schools and academic proficiency test as research tools, and investigated 17,335 fourth-grade students in Q city. The results showed that: (1) Compared with other students, students with poor academic performance scored lower in interpersonal relationship, among which the score of peer relationship was the lowest, and the score of girls was significantly higher than that of boys; (2) There is a significant positive correlation between the interpersonal relationship and the academic performance of the students with poor academic performance, which has a predictive effect on the academic performance, and the teacher-student relationship has the highest predictive rate on the academic performance. In order to improve the academic performance of students with academic disabilities and promote their physical and mental health development, parents and schools should pay attention to the interpersonal relationship of students with academic disabilities and cultivate students' social communication skills through diversified ways. To improve teachers' sense of responsibility and strengthen the transformation of students with poor academic performance; Work together to create a good interpersonal atmosphere to promote the all-round development of students.
\end{abstract}

Keywords: Poor Academic Performance, Interpersonal Relationship, Mathematics Achievement

\section{学业不良学生人际关系与数学成绩的关系研究}

\author{
郑皓月，栾晓姣，王海涛* \\ 中国海洋大学基础教学中心, 青岛, 中国
}

邮箱

793744056@qq.com（郑皓月），1982824256@qq.com（栾晓姣）, yshdwht@163.com（王海涛）

摘要: 人际关系已经成为学业不良学生社会性研究的一个重要方面,因为人际关系不但对学业不良学生的学业发展产生 影响,而且还会引发一系列心理问题, 因此进行这方面的探讨具有十分重要理论和实践价值。为探究学业不良学生人际 关系与数学成绩的关系, 本研究采用整群随机抽样的方法, 以中小学人际关系量表、学业水平测试为研究工具, 对 $Q$ 市17335名四年级学生进行调查研究。研究结果表明: (1) 与其他学生相比, 学业不良的学生人际关系得分较低, 其 
中同伴关系得分最低, 女生得分显著高于男生; (2) 学业不良学生人际关系与学业成绩呈显著正相关, 对学业成绩具 有预测作用, 其中师生关系对学业成绩预测率最高。为提升学业不良学生的学业成绩, 促进学生的身心健康发展, 家 长和学校都应该关注学业不良学生的人际关系, 通过多元化方式培养学生社会交往技能; 提高教师责任心, 加强对学 业不良学生的转化; 共同营造良好的人际关系氛围，促进学生全面发展。

关键词: 学业不良, 人际关系, 数学成绩

\section{1. 引言}

2010年上半年，我国颁布《国家中长期教育改革和发 展规划纲要（2010-2020）》, 明确提出要“关心每个学生, 促进每个学生主动地、生动活泼地发展, 为每个学生提供 适合的教育”、“建立学习困难学生的帮助机制”。学业不 良的现象在各个学段中广泛存在, 不仅会影响学生学习热 情, 还严重地阻碍了我国教育事业发展, 降低了教育效益 [1]。因此, 关注并分析学业不良问题, 提出有效建议, 促 进每一个学生发展是至关重要的。

人际关系是指人与人之间通过交往与相互作用而形成 的直接的心理关系, 是个体社会化重要的组成部分。生态系 统理论认为, 一个人成长的环境分为宏观系统、外观系统、 中观系统、微观系统、时间系统五方面[2], 其中微观系统对 学生产生直接影响, 包括学生的家庭和学校, 主要是与父母、 教师、同伴进行互动。已有研究表明, 良好的人际关系能帮 助学业不良的学生获得更多学业上的支持 [3-4], 建立积极的 学习态度, 从而对学业成绩起到促进作用。

一直以来, 小学生的学业表现、人际关系、心理健康 等问题都受到广大一线教师以及教育研究者的关注, 国内 有大量关于人际关系、学业成就的研究, 但是针对小学生 数学学业不良学生的人际关系研究少之又少。数学是一切 科学的基础, 也是学校教育中不可缺少的一部分, 小学阶 段的数学学习大都是以基础知识为主, 数学学业水平低不 仅会导致学生产生消极的学业情绪, 还会影响后续的数学 学习[5]。因此, 本研究以小学四年级学生为研究对象, 从 人际关系角度出发, 了解和把握学业不良学生的现状, 分 析人际关系对其学业表现的影响, 为教师和家长有效改善 小学数学学业不良成绩提供一定的指导。

\section{2. 研究过程及结果分析}

\section{1. 研究对象}

采取整群随机取样的方法对Q市17335名四年级学生 进行问卷调查和学业测试, 发放问卷和试卷17355份, 问 卷回收率为 $100.0 \%$ 。其中回收问卷中有效问卷 17099 份, 有效率为 $98.52 \%$ ，男生 8839 名 $(51.7 \%)$ ，女生 8260 名 $(48.3 \%) ，$ 平均年龄为 9.80 岁 $(\mathrm{SD}=0.44)$ 。

\section{2. 研究工具}

\subsection{1. 中小学人际关系量表}

本研究采用“Q市教育质量监测项目”编制的“中小学 人际关系量表”。该量表包括 10 道题目, 分为亲子关系、
师生关系和同伴关系 3 个维度, 采用 5 点计分, 分别是很不 符合、不太符合、不确定、比较符合、非常符合, 记为 $1-5$ 分, 得分越高说明学生的人际关系越好。量表的内部一致 性系数 $\alpha=0.648$ 。

\subsection{2. 学业水平测试工具}

本研究采用“Q市教育质量监测项目”中的数学学业水 平测试工具, 依据《义务教育数学课程标准》设计相关内 容。该工具内部一致性系数 $0.769-0.837$, 采取选择和解答 两种题型, 选择题全部为 4 选 1 的单项选择题, 采用 $0 、 1$ 方式计分, 解答题要求被试列出相应的解题步骤, 采用多 级计分方式。

\section{3. 调查方法与统计分析}

\subsection{1. 调查方法}

问卷调查部分全部采用网络系统平台进行数据采集, 学业水平测试采用纸笔作答的方式, 由经过统一培训的调 查员, 要求被试在规定的时间内按照统一指导语完成网络 问卷和学业水平试卷, 网络问卷直接保存于网络平台中, 学业水平试卷当场收回。

\subsection{2. 统计分析}

问卷和学业水平试卷统一编码存储, 其中学业水平试 卷由教育测量专家、数学学科专家、一线教师组成水平标 准划定小组, 根据安戈夫法对其数学学业成绩进行标准划 定, 将其划分为四个水平: 不合格、合格、良好、优秀。 其本研究将水平不合格的界定为学业不良。

运用SPSS22.0统计软件包进行统计处理, 使用均数、 标准差、相关分析和回归分析等分别对资料进行统计描 述。

\section{4. 调查结果及分析}

调查发现, 按照安戈夫法对学生数学学业成绩进行的 标准划定, 学业不良的学生共 2414 名, 其中男生1320人, 女生1094人。

\subsection{1. 学生的人际关系得分}

本研究发现, 学生在人际关系总得分为 41.49 , 其中 亲子关系得分最高 $(M=16.18)$, 同伴关系得分最低 $(M=11.50)$ 。与其他学生相比, 学业不良的学生人际关系 总得分最低 $(M=38.23)$, 其中亲子关系得分最高 $(M=15.10)$, 同伴关系得分最低 $(M=10.03)$, 具体结果见表 1 。 
表1 学生的人际关系得分。

\begin{tabular}{llllll}
\hline 人际关系 & 不合格 & 合格 & 良好 & 优秀 & 总计 \\
\hline 亲子 & 15.10 & 16.06 & 16.61 & 16.93 & 16.18 \\
师生 & 13.09 & 13.81 & 14.04 & 14.14 & 13.81 \\
同伴 & 10.03 & 11.32 & 12.06 & 12.68 & 11.50 \\
总维度 & 38.23 & 41.19 & 42.71 & 43.76 & 41.49 \\
\hline
\end{tabular}

\subsection{2. 学业不良的男、女学生在人际关系上的性别差异}

数据统计分析发现（见表2），学业不良的男、女学生人 际关系得分上的差异有统计学意义 $(\mathrm{P}<0.01)$, 男生总得分 低于女生 $(\mathrm{M}=37.53<\mathrm{M}=39.06)$ 。从各维度得分来看, 学业不良 的四年级男、女生在亲子关系、师生关系和同伴关系的得分 差异都有统计学意义 $(\mathrm{P}<0.01)$, 均表现为男生得分低于女生。

表2 学业不良的男、女学生在人际关系上的性别差异。

\begin{tabular}{lllllll}
\hline 人际 & \multicolumn{9}{l}{ 男 $(\mathbf{n 1 = 1 3 2 0})$} & \multicolumn{2}{l}{ 女 $(\mathbf{n 2}=\mathbf{1 0 9 4})$} & \multirow{2}{*}{$\mathbf{~}$} & \multirow{2}{*}{$\mathbf{p}$} \\
\cline { 2 - 5 } 关系 & $\mathbf{M}$ & $\mathbf{S D}$ & $\mathbf{M}$ & $\mathbf{S D}$ & & \\
\hline 亲子 & 14.94 & 2.66 & 15.29 & 2.69 & -3.155 & 0.002 \\
师生 & 12.96 & 2.76 & 13.25 & 2.46 & -2.712 & 0.007 \\
同伴 & 9.63 & 2.77 & 10.52 & 2.83 & -7.891 & 0.000 \\
总分 & 37.53 & 5.82 & 39.06 & 5.90 & -6.390 & 0.000 \\
\hline
\end{tabular}

\subsection{3. 学生的人际关系与数学成绩的相关分析}

通过Pearson相关分析发现（见表3），各个维度、总 维度与学业不良学生成就均成显著正相关。数学学业不良 的学生成就与人际关系总维度的相关系数最高 $(\mathrm{r}=0.232$, $\mathrm{P}<0.001$ ), 其中师生关系与学业总分相关系数最高 $(\mathrm{r}=0.187, \mathrm{P}<0.001)$ 。

表3 学业不良学生的人际关系与数学成绩的相关分析。

\begin{tabular}{lllll}
\hline 人际关系 & 不合格 & 合格 & 良好 & 优秀 \\
\hline 亲子关系 & $.167^{* *}$ & $.074^{* *}$ & $.054^{* *}$ & 0.021 \\
师生关系 & $.187^{* *}$ & $.055^{* *}$ & 0.020 & 0.040 \\
同伴关系 & $.154^{* *}$ & $.109^{* *}$ & $.064^{* *}$ & 0.017 \\
总维度 & $.232^{* *}$ & $.110^{* *}$ & $.062^{* *}$ & 0.032 \\
\hline
\end{tabular}

\subsection{4. 学业不良学生人际关系与数学成绩的回归分析}

为了进一步说明人际关系对学业不良学生数学成绩 的影响程度（见表4），我们在控制性别因素后建立了师 生、亲子和同伴关系对学业不良学生数学成绩的多元回归 模型。结果显示, 三种人际关系对学业不良学生数学成绩 的解释率为 $5.7 \%$, 其中师生关系解释率最高。

表4 学业不良学生人际关系与数学成绩的回归分析。

\begin{tabular}{|c|c|c|c|c|c|}
\hline \multirow{2}{*}{ 模型 } & \multicolumn{2}{|c|}{ 非标准化系数 } & \multirow{2}{*}{$\begin{array}{l}\text { 标准系数 } \\
\text { Beta }\end{array}$} & \multirow[b]{2}{*}{$\mathrm{t}$} & \multirow{2}{*}{ Sig. } \\
\hline & B & 标准误差 & & & \\
\hline 常数 & 234.152 & 7.859 & & 29.796 & 0.000 \\
\hline 性别 & 7.165 & 2.406 & 0.060 & 2.978 & 0.003 \\
\hline 亲子关系 & 1.686 & 0.508 & 0.075 & 3.319 & 0.001 \\
\hline 师生关系 & 3.000 & 0.505 & 0.132 & 5.945 & 0.000 \\
\hline 同伴关系 & 2.195 & 0.441 & 0.103 & 4.976 & 0.000 \\
\hline
\end{tabular}

\section{3. 研究结论}

本研究通过对 $\mathrm{Q}$ 市小学四年级学业不良学生进行的调 查研究与分析, 可以得出以下结论:
第一, 与其他学生相比, 学业不良的学生人际关系得 分较低, 其中师生关系、同伴关系得分较低。原因可能是 学业不良儿童本身因为学业成绩长期处于落后地位[6-7], 被学校、社会、同伴贴上差生的标签, 受到比其他人更多 的冷落、讽刺、批评, 因此与父母、教师、同学关系不够 理想[8]。由于学业不良学生往往会伴随一些问题行为, 教 师需要花更多的时间和精力管理学业不良学生。同时学业 不良学生存在较大的学业和人际压力, 与同龄人交往时往 往会出现摩擦的情况, 发生一些不愉快, 与教师、同学的 交往中会发生更多的不良体验。

第二, 学业不良女生的人际交往水平高于学业不良男 生, 这与以往研究结果一致。沃建中等人认为男女生生理 发育的时间差异和不同性格特点是造成女生人际关系好 于男生的原因, 由于女生比男生更早进入青春期, 心理上 的成熟也早于男生, 语言发展较好, 表达更加清晰流畅, 在友好相处能力和冲突平息能力方面比男生强[9]。根据社 会心理学的有关理论研究, 女生受到生理遗传因素、社会 文化因素、家庭环境因素、自然环境等各种因素的影响, 女生比男生情感更为细淢, 在情绪表达上比男生更具优 势, 并且社会文化也鼓励女性表达自己的情感, 因此在人 际关系水平上，女生要优于男生。

第三, 学业不良学生的学业成就与人际关系有更高的 相关度, 而人际关系对成绩优秀的学生影响不具有显著性 差异。一个可能的原因在于, 成绩优秀的学生在学习方面 有很大的自主性, 学习动机更多的是来自自身需求, 具有 更高的自我效能感。已有研究发现学业不良的学生与其他 学生相比, 自我效能感较低, 而低自我效能感的学生在学 习中是被动的 [10]。因此, 在日常的学习中学业不良的学 生学习动机依赖于其他人的激励或支持, 表现为与父母的 关系以及学校中师生及同学之间的相互关系[11]。表1结果 显示学业不良的学生与父母、教师和同伴的交往并不够顺 利, 也可能是干扰学业不良学生学业表现的原因之一。

第四, 师生关系对学业不良学生学业成绩的影响最 高。首先, 可能的原因是与其他学生相比, 学业不良学生 更加在乎教师对自己的情感、期望与评价。已有研究证明 良好的师生关系能够促进学生的学业成绩[12], 教师与学 生互动过程中更容易发现学业不良学生学习过程中碰到 哪些困难, 精准地为学生提供学习支持[13]。其次, 教师 在班级教学活动和日常活动中, 都能够公平地对待学生, 不带“有色眼镜”来看待学业不良学生, 给予学生温暖与力 量[14]。因此师生关系对学业不良学生成绩影响更大。

\section{4. 思考与建议}

\section{1. 关注学业不良学生的人际关系, 培养学生社会交往 技能}

提升学业不良学生的社会交往能力有助于促进学生成 就。首先, 家长作为孩子的第一任老师, 应主动挑起教育 孩子的重任, 在日常生活中教给孩子交往技巧, 从而帮助 孩子拥有良好的校园关系, 助力学生学业发展 [15]。其次, 学校可以开发交往相关的校本课程[16], 针对本校学业不 良、人际交往所存在的问题, 设计有针对性的、合适的主 
题课程, 以学校课程为保障来审视并解决学业不良学生的 人际交往问题; 最后, 教师在课堂或活动中采用角色扮演 或换位思考的方法帮助儿童除去自我中心的思考方式, 学 会理解别人, 学会理解交往对象的真正意图。其中需要注 意的是, 教师需要学习个性化辅导, 面对不同的个体类别 例如男生、女生, 要理解其中男、女在生理因素、情感表 达、人际交往中的不同，从而进行个性化的心理教育[17]。

\section{2. 提高教师责任心, 加强对学业不良学生的转化}

教师在学业不良学生的学习中占据关键作用, 是影响 学生学业成绩的主要因素。教师应该增强责任心, 承担起 应有的责任, 帮助学业不良学生拥有良好的人际关系, 改 善自己的学业表现。作为教师自身 [18], 第一, 教师应在 保证与学生关系融洽的同时改进自己的教学方式, 依据讲 授的内容和学生的年龄特点选用与之相适应的新型教学 法, 调动学生学习热情; 第二, 教师应该根据学业不良学 生的基础水平、学习策略等方面的具体情况,把学生分层, 精准地对学生提出作业或任务要求并给予有效的帮助; 第 三, 在班集体学习小组活动时, 教师可以根据合作学习、 分组学习、项目式学习等方式, 使学业不良儿童有机会与 班级中优秀同学共同学习和交流[19], 促进学业不良学生 转变。第四, 教师应该引导父母成为教育的促进力量。教 师可以通过家长会、家访随时与家长进行交流和沟通, 了 解学生的成长背景、交友情况等多方面信息, 与家长一起 分析学生学业不良的原因。

\section{3. 多方共同营造良好的人际关系氛围, 促进学生学业 发展}

孩子的教育工作需要家长、学校和学生三方的共同努 力才能完成。首先, 学校应该改进家校沟通的途径与方式, 加强家庭与学校的联系, 实现家校之间的双向信息沟通 [20]。关注学业不良学生的心理健康与学业表现, 及时发 现问题进行干预, 给予学生温暖与支持, 有助于预防学生 心理和学业问题。其次, 学校应该定期组织讲座或报告, 指导家长科学地进行家庭教育, 提高家长对子女的管理水 平。尤其对于学业不良学生的家长, 应该开展有针对性的 主题活动帮助家长了解学业不良学生的心理, 从而有效地 改善学生的心理健康与学业表现。

\section{参考文献}

[1] 孟海霞.小学生学业成绩不良成因及对策研究 [J].科学咨询 (科技·管理),2019(07):96.

[2] Kung H Y, Lee C Y. Factors Influencing Junior High School Students' English Language Achievement in Taiwan: A Bronfenbrenner's Ecological System Approach[J]. Journal of Educational Practice and Research, 2016.
[3] Kiuru N, Wang M T, Salmela-Aro K, et al. Associations between Adolescents' Interpersonal Relationships, School Well-being, and Academic Achievement during Educational Transitions[J]. Journal of Youth and Adolescence, 2020, 49(Supplement 2).

[4] Martin A J, Dowson M. Interpersonal Relationships, Motivation, Engagement, and Achievement: Yields for Theory, Current Issues, and Educational Practice[J]. Review of Educational Research, 2009, 79(1):327-365.

[5] 蔡丹,李其维,邓赐平.数学学业不良初中生的工作记忆特点: 领域普遍性还是特殊性?[J].心理学报,2013,45(02):193-205.

[6] 桑青松. 非智力因素造成学业不良学生的学习心理辅导 [J]. 中国教育学刊,2001(05):38-40.

[7] Woodcock S, Faith E. Am I to blame? Teacher self-efficacy and attributional beliefs towards students with specific learning disabilities[J]. Teacher Development, 202

[8] 沈烈敏.学业不良学生的心理弹性研究初探 [J]. 心理科 学,2009,32(05):1189-1191+1161.

[9] 沃建中, 林崇德, 马红中, 李峰.中学生人际关系发展特点的研 究[J].心理发展与教育,2001(03):9-15.

[10] 刘伟芳,葛明贵.学业不良学生学习效能感及其发展 [J].教育 研究与实验,2005(02):61-64.

[11] 王根顺,周晓玉.谈学业不良学生学习动机的补救[J].中国电 力教育,2010(24):178-180.

[12] 周文叶,边国霞,文艺.师生关系对学生学业成绩的影响 基于师生关系与学业成绩关系的实证研究综述 [J]. 外国教 育研究,2020,47(09):18-34.

[13] 张云运,刘思辰,任萍,牛丽丽.学生学业和行为特征如何影响 教师支持? 来自个体和圈子层面的证据 [J]. 心理发展与教 育,2020,36(03):318-328.

[14] 张秀阁,梁宝勇,藏丽荣,马小兰.学习优秀与学习困难初二学 生的内隐完美态度研究[J].中国学校卫生,2007(07):624-625.

[15] 谷长芬,张庆平. 父母教养方式对小学学业不良儿童孤独感 的影响[J].中国心理卫生杂志,2008(03):179-182.

[16] 叶小耀. 初中校本课程的开发与实践研究 $[J]$. 河南教育 (上 旬), 2014, 000(011):23-24.

[17] Nind M. A question of perspective-parenting and other matters in the lives of people with learning disabilities[J]. British Journal of Learning Disabilities, 2021, 49(1):1-2.

[18] 王关群.中学生学业不良的成因及对策探析 [J].基础教育参 考,2010(17):20-22.

[19] 王海燕.团体心理辅导对学业不良初中生学业求助的影响 [J].中国临床心理学杂志,2012,20(03):420-423.

[20] 韩洪波.从形式到实质: 在线教学中家校合作的策略[J].教育 理论与实践,2020,40(29):52-55. 